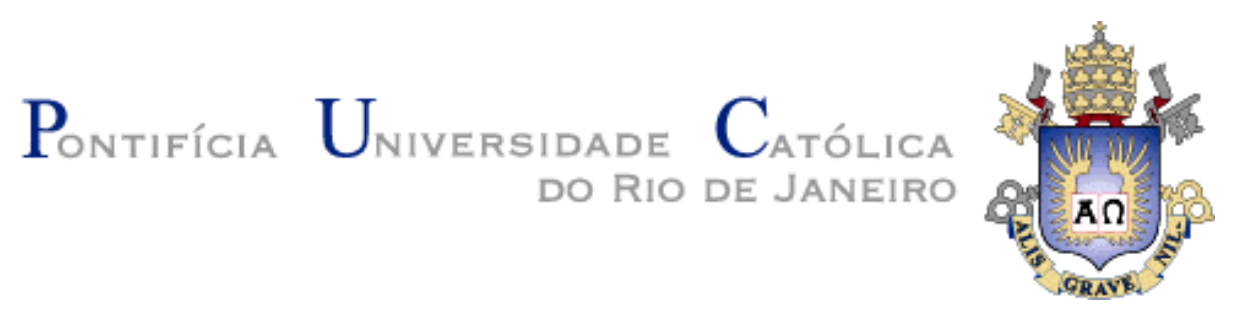

Fernando de Freitas Silva

\title{
Uma nova abordagem de mineração de repositórios de software utilizando ferramentas da Web Semântica
}

Dissertação de Mestrado

Dissertação apresentada como requisito parcial para obtenção do grau de Mestre pelo Programa de Pósgraduação de Informática da PUC-Rio.

Orientador: Prof. Daniel Schwabe

Rio de Janeiro

Agosto de 2013 


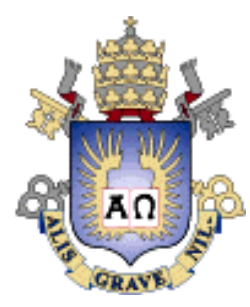

Fernando de Freitas Silva

\section{Uma nova abordagem de mineração de repositórios de software utilizando ferramentas da Web Semântica}

Dissertação apresentada como requisito parcial para obtenção do título de Mestre pelo Programa de PósGraduação em Informática da PUC-Rio. Aprovada pela Comissão Examinadora abaixo assinada.

Prof. Daniel Schwabe Orientador

Departamento de Informática - PUC-Rio

Prof. Arndt Von Staa

Departamento de Informática - PUC-Rio

Prof. Alessandro Garcia

Departamento de Informática - PUC-Rio

Prof. José Eugenio Leal

Coordenador(a) Setorial do Centro Técnico Científico - PUC-Rio

Rio de Janeiro, 15 de agosto de 2013 
Todos os direitos reservados. É proibida a reprodução total ou parcial do trabalho sem autorização da universidade, do autor e do orientador.

\section{Fernando de Freitas Silva}

Graduou-se em Bacharel em Sistemas de Informação pela Pontifícia Universidade Católica do Rio de Janeiro em 2009.

Ficha Catalográfica

Silva, Fernando de Freitas

Uma nova abordagem de mineração de repositórios de software utilizando ferramentas da Web semântica / Fernando de Freitas Silva ; orientador: Daniel Schwabe. - 2013.

$178 f$ : il. ; $30 \mathrm{~cm}$

Dissertação (mestrado)-Pontifícia Universidade Católica do Rio de Janeiro, Departamento de Informática, 2013.

Inclui bibliografia

Informática - Teses. 2. Web semântica. 3.

Manutenção de software. 4. Engenharia de software. 5. Semântica. 6. Repositórios de software. I. Schwabe, Daniel. II. Pontifícia Universidade Católica do Rio de Janeiro. Departamento de Informática. III. Título. 


\section{Agradecimentos}

Agradeço aos meus pais, Walquíria de Freitas Silva e Fernando Jorge Lopes Silva, por sempre me apoiarem, incentivarem e por terem me dado uma criação excepcional. Sem vocês eu não estaria onde estou hoje. Muito obrigado!

Agradeço a minha esposa e melhor amiga, Geyzyely, pelo apoio durante esta empreitada e por ter compreendido as madrugadas que tive que passar longe de você para estudar. Obrigado por estar ao meu lado e espero que eu possa te apoiar do mesmo modo que você me apoiou. Te amo.

Agradeço ao meu "amigão" e filho, Joaquim, que apesar de sua pouca idade entendia os momentos que eu não podia brincar ou estar com ele. Obrigado por ser um filho maravilhoso e que você alcance coisas maiores do que eu já imaginei.

Tenho muito a agradecer ao meu orientador Professor Daniel Schwabe pelo constante incentivo e apoio durante Mestrado. Sem suas observações e direcionamentos eu não teria chegado até aqui. Muito obrigado!

Agradeço ao Gustavo Robichez de Carvalho e ao Professor Carlos José Pereira de Lucena pelo apoio durante minha graduação e pela oportunidade de ingressar no Mestrado. Muito obrigado!

Agradeço aos meus colegas da PrimeUp, ao sócio Leandro Daflon e ao meu gerente João Manoel Silvestre pelo compreensão nos momentos que tive que me ausentar do trabalho durante meu Mestrado. Muito obrigado!

Agradeço à PUC-Rio que me formou Bacharel em Sistemas de Informação e me deu a oportunidade de me tornar Mestre em Informática sem custo algum.

Finalmente, gostaria de agradecer à CAPES pela bolsa concedida durante os dois anos do Mestrado. Este apoio foi imprescindível para que eu pudesse concluir meus estudos. Obrigado! 


\section{Resumo}

Silva, Fernando de Freitas; Schwabe, Daniel. Uma nova abordagem de mineração de repositórios de software utilizando ferramentas da Web Semântica. Rio de Janeiro, 2013. 178p. Dissertação de Mestrado Departamento de Informática, Pontifícia Universidade Católica do Rio de Janeiro.

A Mineração de Repositórios de Software é um campo de pesquisa que extrai e analisa informações disponíveis em repositórios de software, como sistemas de controle de versão e gerenciadores de issues. Atualmente, diversos trabalhos nesta área de pesquisa têm utilizado as ferramentas da Web Semântica durante o processo de extração a fim de superar algumas limitações que as abordagens tradicionais enfrentam. O objetivo deste trabalho é estender estas abordagens que utilizam a Web Semântica para minerar informações não consideradas atualmente. Uma destas informações é o relacionamento existente entre as revisões do controle de versão e as mudanças que ocorrem no Abstract Syntax Trees dos arquivos modificados por essas revisões. Adicionalmente, esta nova abordagem também permite modelar a interdependência entre os projetos de software, suas licenças e extrair informações dos builds gerados por ferramentas de integração contínua. A validação desta nova abordagem é demonstrada através de um conjunto de questões que são feitas por desenvolvedores e gerentes durante a execução de um projeto e que foram identificadas em vários trabalhos da literatura. Demonstramos como estas questões foram convertidas para consultas SPARQL e como este trabalho consegue responder às questões que não são respondidas ou são respondidas parcialmente em outras ferramentas.

\section{Palavras-chave}

Web Semântica; Manutenção de Software; Engenharia de Software Semântica; Repositórios de Software. 


\section{Abstract}

Silva, Fernando de Freitas; Schwabe, Daniel (Advisor). A New Approach for Mining Software Repositories using Semantic Web Tools. Rio de Janeiro, 2013. 178p. MSc. Dissertation - Departamento de Informática, Pontifícia Universidade Católica do Rio de Janeiro.

The Mining of Software Repositories is a field of research that extracts and analyzes information available in software repositories, such as version control systems and issue trackers. Currently, several research works in this area have used Semantic Web tools during the extraction process to overcome some limitations that traditional approaches face. The objective of this work is to extend the existing approaches that use Semantic Web tools to mine information not considered in these works. The objective of this work is to extend these approaches using the Semantic Web to mine information not currently considered. One of these information is the relationship between revisions of version control and the changes that occur in the Abstract Syntax Trees of files modified by these revisions. Additionally, this new approach also allows modeling the interdependence of software projects, their licenses and extracting information from builds generated by continuous integration tools. The validation of this approach is demonstrated through a set of questions that are asked by developers and managers during the execution of a project and have been identified in various works in the literature. We show how these questions were translated into SPARQL queries and how this work can answer the questions that are not answered or are partially answered in other tools.

\section{Keywords}

Semantic Web; Software Maintenance; Semantic Software Engineering; Software Repositories. 


\section{Sumário}

1 Introdução 18

1.1. Limitações das Abordagens Atuais 21

1.2. Objetivo 22

1.3. Organização da Dissertação 24

2 Fundamentos $\quad 26$

2.1. Visão Geral das Ferramentas utilizadas no Ciclo de Vida de Desenvolvimento de Software 26

2.2. Tecnologias da Web Semântica 29

2.2.1. Resource Description Framework (RDF) 30

2.2.2. RDF Schema (RDFS) 32

$\begin{array}{ll}\text { 2.2.3. SPARQL } & 34\end{array}$

2.2.4. Web Ontology Language (OWL) 36

3 Ontologias e Vocabulários $\quad 39$

3.1. Description of a Project (DOAP) 39

3.2. Friend of a Friend (FOAF) 40

3.3. Dublin Core 42

3.4. Creative Commons Rights Expression Language (CC Rel) 43

3.5. Open Services for Lifecycle Collaboration (OSLC) 44

3.5.1. OSLC Change Management Vocabulary (OSLC CM) 45

3.5.2. OSLC Automation Management Vocabulary (OSLC Auto) 47

3.5.3. OSLC Asset Management Vocabulary (OSLC Asset) 48

3.5.4. OSLC Quality Management Vocabulary (OSLC QM) 50

3.5.5. OSLC Software Configuration Management Vocabulary (OSLC SCM) 51

3.5.6. As Novas Ontologias 52

4 A Plataforma de Extração 63

4.1. Casos de Uso da Plataforma 63

4.1.1. Cadastrar Requisição de Extração de Informações de Controle de Versão

4.1.2. Cadastrar Requisição de Extração de Informações de Demandas e 
Defeitos

4.1.3. Cadastrar Requisição de Extração de Informações de Integração

Contínua

4.1.4. Cadastrar Requisição de Extração de Informações de Versão de uma

Biblioteca

65

4.1.5. Cadastrar Requisitar de Extração de Diferenças entre Versões de uma Biblioteca

4.1.6. Executar Consulta SPARQL

66

4.1.7. Pesquisar Requisições de Extração

66

4.1.8. Iniciar Requisição de Extração

66

4.2. Arquitetura da Plataforma

67

4.3. Arquitetura de Implementação

75

4.3.1. Tecnologias de Infraestrutura

75

4.3.2. Tecnologias para a persistência dos Dados RDF 83

4.3.3. Tecnologias de Apoio aos Conectores

84

5 Validação e Estudo de Caso

88

5.1. Introdução

88

5.2. Perguntas Frequentes

89

5.3. Exemplos de Novas Perguntas

94

5.4. Estudos de Caso

97

6 Conclusão e Trabalhos Futuros

104

6.1. Trabalhos Relacionados

104

6.2. Contribuições

105

6.3. Trabalhos Futuros

106

7 Referências Bibliográficas

108

Apêndice A - Módulos Conectores Comuns

Apêndice B - Módulos Transformadores RDF

Apêndice C - Módulos Auxiliares 
Apêndice E - Consultas SPARQL "Perguntas Frequentes dos

Desenvolvedores" 


\section{Lista de figuras}

Figura 1 Exemplo do Fluxo de Informações entre ferramentas utilizadas no desenvolvimento de software.

Figura 2 Exemplos de Representação de uma mesma informação nas

três modelagens .....

Figura 3 Exemplo da Notação RDF/XML representando as informações

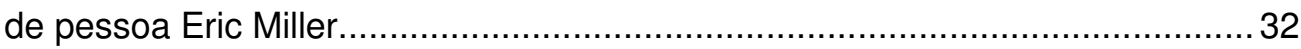

Figura 4 Exemplo de uma consulta SPARQL do tipo SELECT .......................... 35

Figura 5 Exemplo da representação das informações de um projeto

utilizando DOAP

Figura 6 Exemplo da representação de informações utilizando a ontologia

FOAF

Figura 7 Representação das informações de uma licença utilizando

o vocabulário CC REL

Figura 8 llustração da sinergia de ferramentas aderentes aos padrões da

OSLC.

Figura 9 Representação das informações de um defeito utilizando o

vocabulário OSLC CM

Figura 10 Representação do resultado de uma automação utilizando

o vocabulário OSLC Automation.

Figura 11 Exemplo de Representação das informações de um ativo utilizando

o vocabulário OSLC Asset.

Figura 12 Exemplo da representação de um Caso de Teste utilizando

o vocabulário OSLC Quality Management

Figura 13 Exemplo de representação de uma classe

chamada "org.example.Class1" na ontologia Evoont SOM e na

ontologia EPR

Figura 14 Exemplo de representação do relacionamento entre um método e

o tipo de seu retorno

$\mathrm{Na}$ tabela abaixo apresentamos os principais conceitos e propriedades que são definidas nesta ontologia. Já na Figura 15, demonstramos visualmente como estes principais conceitos se relacionam.

Figura 15 Representação visual dos elementos da ontologia de

Entidades, Propriedades e Relacionamentos 
Figura 16 Representação da exclusão de um parâmetro de um método na ontologia EPR

Figura 17 Representação da relação de membro entre a pessoa "p1" e o comitê "c1"......

Figura 18 Representação da nova entidade "membership" que foi criada para representar informações sobre o relacionamento da pessoa "p1" e o comitê "c1"

Figura 19 Representação do responsável pela nomeação da pessoa "p1"

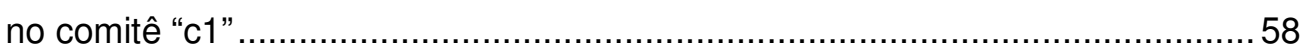

Figura 20 Exemplo da Hierarquia de impactos.

Figura 21 Representação da modificação da visibilidade de um método de "package" para "public" 62

Figura 22 Diagrama de Casos de Uso da Plataforma 64

Figura 23 Visão Geral dos Processos de Extração implementados na

Plataforma de Extração

Figura 24 Visão Geral do Processo de Extração de Informações de Ferramentas de Controle de Versão.

Figura 25 Visão Geral do Processo de Extração de Informações de uma Versão de uma Biblioteca

Figura 26 Visão Geral do Processo de Extração de Informações de

Ferramentas de Integração Contínua 73

Figura 27 Visão Geral do Processo de Extração de Informações de

Diferenças entre duas Versões de uma Biblioteca.

Figura 28 Visão Geral do Processo de Extração de Informações de

Ferramentas de Gerenciamento de Demandas/Defeitos 75

Figura 29 Arquitetura definida pela especificação OSGi .77

Figura 30 Interfaces que o módulo Conector de Ferramentas de

Controle de Versão fornece .78

Figura 31 Exemplo de um MANIFEST.MF de um bundle 78

Figura 32 Exemplo da dinâmica entre dois bundles e um serviço fornecido por um deles. 79

Figura 33 Código que registra novos serviços do tipo ISCMConnector.... 80

Figura 34 Código que recebe notificação da remoção de um serviço do tipo ISCMConnector e remove do SCMConnectorRegister. 81

Figura 35 Trecho do Arquivo XML de Configuração do serviço fornecido pelo Módulo Conector Git 82 
Figura 36 Parte dos Atores criados para o processo de Extração de Informações de Ferramentas de Controle de Versão.

Figura 37 Visualização das Chamadas de um Método na IDE Eclipse

Figura 38 Visualização de todos os defeitos abertos e sem um responsável na ferramenta Atlassian Jira

Figura 39 Imagem do GitHub que demonstra o desenvolvedor Sean Owen como o desenvolvedor com maior número de commits no período de tempo....999

Figura 40 Tabela com os métodos mais invocados no projeto. 101

Figura 41 Imagem da Página do GitHub que indica os dois desenvolvedores retornados pela consulta como os principais contribuidores da classe 102

Figura 42 Interface ISCMConnector

Figura 43 A classe SCMConnectorRegister e a interface

ISCMConnectorRegister 112

Figura 44 Diagrama de Estrutura Composta do Módulo Conector de

Ferramenta de Controle de Versão

Figura 45 Diagrama de Sequência da integração entre o cliente o

Módulo Conector de Ferramenta de Controle de Versão 113

Figura 46 Diagrama de Estrutura Composta do Módulo Conector Git..... 114

Figura 47 Interface ICIConnector 114

Figura 48 A classe CIConnectorRegister e a interface ICIConnectorRegister .. 115 Figura 49 Diagrama de Estrutura Composta do Módulo Connector Comum de Ferramentas de Integração Contínua. 116

Figura 50 Diagrama de Sequência da integração entre o cliente o Módulo Connector de Ferramentas de Integração Contínua. 117

Figura 51 Diagrama de Estrutura Composta do Módulo Conector Jenkins 118

Figura 52 Interface IAssetConnector 118

Figura 53 A classe AssetConnectorRegister e a interface IAssetConnectorRegister..... 119

Figura 54 Diagrama de Estrutura Composta do Módulo Connector de Ferramentas de Gerenciamento de Artefatos 120

Figura 55 Diagrama de Sequência da integração entre o cliente o

Módulo Connector de Ferramentas de Gerenciamento de Artefatos. 120

Figura 56 Diagrama de Estrutura Composta do Módulo Conector Aether 121

Figura 57 Interface ICMConnector 121

Figura 58 A classe CMConnectorRegister e a interface 
Figura 59 Diagrama de Estrutura Composta do Módulo Conector Comum de Ferramentas de Gerenciamento de Defeitos e Demandas.

Figura 60 Diagrama de Sequência da integração entre o cliente o

Módulo Conector de Ferramentas de Gerenciamento de Demandas e

Defeitos 123

Figura 61 Diagrama de Estrutura Composta do Módulo Conector Jira 124

Figura 62 Interface IASTConnector. 125

Figura 63 A classe ASTConnectorRegister e a interface

IASTConnectorRegister 125

Figura 64 Diagrama de Estrutura Composta do Módulo Conector Comum de Linguagem de Programação 126

Figura 65 Diagrama de Sequência da integração entre o cliente o

Módulo Conector de Linguagem de Programação. 126

Figura 66 Diagrama de Estrutura Composta do Módulo Conector de

Linguagem de Programação Java

Figura 67 Interface IDependencyConnector.

Figura 68 A classe DependencyConnectorRegister e a interface

IDependencyConnectorRegister 128

Figura 69 Diagrama de Estrutura Composta do Módulo Comum Conector de Ferramentas de Gerenciamento de Dependências 129

Figura 70 Diagrama de Sequência da integração entre o cliente o

Módulo Conector de Ferramentas de Gerenciamento de Dependência...... 130

Figura 71 Diagrama de Estrutura Composta do Módulo Conector da

Ferramenta Maven 131

Figura 72 Interface ILicenseConnector 131

Figura 73 A classe LicenseConnectorRegister e a

interface ILicenseConnectorRegister 132

Figura 74 Diagrama de Estrutura Composta do Módulo Comum Connector de Licenças

Figura 75 Diagrama de Sequência da integração entre o cliente o

Módulo Conector de Licenças.

Figura 76 Diagrama de Estrutura Composta do Módulo Conector de Licenças Maven 134

Figura 77 Diagrama de Estrutura Composta do Módulo Transformador RDF de Controle de Versão 135 
Figura 78 Diagrama de Estrutura Composta do Módulo Transformador RDF de Licença.

Figura 79 Diagrama de Estrutura Composta do Módulo Transformador RDF de Artefatos

Figura 80 Diagrama de Estrutura Composta do Módulo Transformador RDF de Código-Fonte.

Figura 81 Diagrama de Estrutura Composta do Módulo Transformador RDF de Impactos

Figura 82 Diagrama de Estrutura Composta do Módulo Transformador RDF de Integração Contínua

Figura 83 Diagrama de Estrutura Composta do Módulo Transformador RDF

de Demandas/Defeitos

Figura 84 Diagrama de Estrutura Composta do Módulo Transformador RDF

de Testes. 149

Figura 85 Diagrama de Estrutura Composta do Módulo FOAF ....................... 150

Figura 86 Diagrama de Estrutura Composta do Módulo DOAP 151

Figura 87 Diagrama de Estrutura Composta do Módulo de Verificação do Tipo de Arquivo. 153

Figura 88 Diagrama de Estrutura Composta do Módulo de Análise de Impactos 155

Figura 89 Diagrama de Componentes dos Módulos utilizados pelo Módulo de Coordenação de Extração de Informações de Ferramentas de Controle de Versão.

Figura 90 Diagrama de Atividades do Processo de Extração de Informações de Ferramentas de Controle de Versão 157

Figura 91 Diagrama de Atividades Tratar Arquivos.... 159

Figura 92 Diagrama de Atividades Tratar Arquivo de Licença. 160

Figura 93 Diagrama de Atividades Tratar Arquivo de Dependências 160

Figura 94 Diagrama de Atividade Tratar Arquivo de Código-Fonte. 161

Figura 95 Diagrama de Atividade Tratar Impactos na AST do Arquivo 162 Figura 96 Diagrama de Componentes dos Módulos utilizados pelo Módulo Coordenador do Processamento da Extração de Informações de uma Versão de uma Biblioteca. 163

Figura 97 Diagrama de Atividades do Processo de Extração de Informações de uma Versão de uma Biblioteca. 163

Figura 98 Diagrama de Componentes dos Módulos utilizados pelo 
Módulo Coordenador de Extração de Informações de Ferramentas

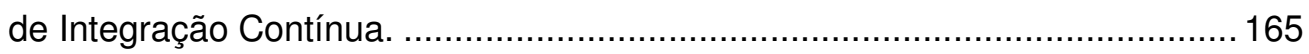

Figura 99 Diagrama de Atividades Extrair Informações de Integração

Contínua. 165

Figura 100 Diagrama de Componentes dos Módulos utilizados pelo Módulo de Coordenação de Extração de Diferenças entre duas Versões de uma Biblioteca.

Figura 101 Diagrama de Atividades do Processo de Extração das diferenças entre duas versões de uma biblioteca.

Figura 102 Diagrama de Componentes dos Módulos utilizados pelo Módulo Coordenador de Extração de Informações de Ferramentas de Gerenciamento de Demandas/Defeitos. 168

Figura 103 Diagrama de Atividades Extrair Informações de Demandas/Defeitos. 169 


\section{Lista de tabelas}

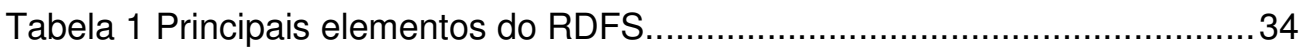

Tabela 2 Elementos do vocabulário DOAP ..................................................... 40

Tabela 4 Principais termos do vocabulário Dublin Core .................................... 43

Tabela 6 Elementos vocabulário OSLC Change Management .........................4 47

Tabela 7 Elementos do vocabulário OSLC Auto ............................................. 48

Tabela 8 Elementos do Vocabulário OSLC Asset Management ........................ 49

Tabela 9 Elementos do vocabulário OSLC Quality Management......................51

Tabela 10 Elementos do vocabulário OSLC SCM........................................... 52

Tabela 11 Principais elementos da ontologia de Entidades, Propriedades

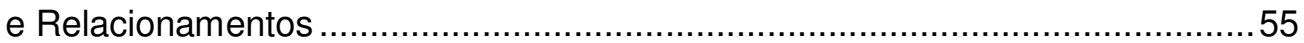

Tabela 12 Principais elementos da ontologia de Impactos ..............................61

Tabela 13 Perguntas Frequentes dos Desenvolvedores ..................................91

Tabela 14 Informações da execução do processo de extração...........................97

Tabela 15 Resultado da Consulta "Quem alterou classes que eu modifico?".....99

Tabela 16 Resultado da Consulta "Quem está utilizando esta API?" para o método "org.apache.mahout.common.AbstractJob.getOption(String)".

Tabela 17 Resultado da Consulta "Quem está utilizando esta API?" para o método "org.apache.mahout.common.AbstractJob.addOption(String)"

Tabela 18 Resultado da Consulta "Para quem atribuir uma revisão de código".....

Tabela 19 Listagem de desenvolvedores que já trabalharam como a biblioteca Lucene. 


\section{Lista de quadros}

Quadro 1 Consulta SPARQL que retornar entidades afetadas pela mudança de versão de uma biblioteca do projeto

Quadro 2 Consulta SPARQL que retorna os elementos afetados por defeitos corrigidos em uma nova versão de uma biblioteca utilizada no projeto .96

Quadro 3 Consulta SPARQL que retorna os elementos que podem ter sido afetados por defeitos devido à atualização da versão de uma biblioteca do projeto.

Quadro 4 Consulta SPARQL que retorna o desenvolvedor que realizou o maior número de modificações nos elementos de código-fonte do projeto .98 Quadro 5 Consulta SPARQL que retorna os dois métodos mais invocados no projeto. 